\title{
Extracts of Koelreuteria henryi Dummer induce apoptosis and autophagy by inhibiting dihydrodiol dehydrogenase, thus enhancing anticancer effects
}

\author{
YUNG-YEN CHIANG ${ }^{1}$, SHU-LI WANG ${ }^{1}$, CHENG-LIN YANG ${ }^{2}$, HUI-YU YANG ${ }^{2}$, HSIU-CHING YANG ${ }^{2}$, \\ J.N. SUDHAKAR ${ }^{2}$, CHING-KUO LEE ${ }^{4}$, HSIU-WEN HUANG ${ }^{5}$, CHIEN-MIN CHEN ${ }^{5}$, SHIOW-HER CHIOU ${ }^{3}$, \\ SHU-FEN CHIANG ${ }^{3}$, HSIN-YUAN FANG ${ }^{6}$, CHIH-YI CHEN ${ }^{6}$, SHWN-HUEY SHIEH $^{7}$ and KUAN-CHIH CHOW ${ }^{2}$ \\ ${ }^{1}$ Department of Dental Laboratory Technology, Central Taiwan University of Science and Technology; \\ ${ }^{2}$ Graduate Institute of Biomedical Sciences, ${ }^{3}$ Graduate Institute of Microbiology and Public Health, \\ National Chung Hsing University, Taichung; ${ }^{4}$ College of Pharmacy, Taipei Medical University, Taipei; \\ ${ }^{5}$ Endemic Species Research Institute, Council of Agriculture, Executive Yuan, Chi-Chi; ${ }^{6}$ Department of Surgery, \\ China Medical University Hospital; ${ }^{7}$ Department of Health Services Administration, \\ China Medical University, Taichung, Taiwan, R.O.C.
}

Received March 12, 2013; Accepted June 7, 2013

DOI: 10.3892/ijmm.2013.1441

\begin{abstract}
Dihydrodiol dehydrogenase (DDH) is frequently detected in cancer cells, and its overexpression correlates with drug resistance, the downregulation of DNA repair mechanisms, increased frequency of tumor recurrence, cancer cell metastasis and poor prognosis. The silencing of DDH expression using siRNA, on the other hand, reduces drug resistance and cancer cell mobility. These data suggest that DDH may be an oncogenerelated protein. However, no specific DDH inhibitor has been identified to date. Thus, in this study, we used DDH as a target enzyme in a live-cell enzyme-linked immunosorbent assay to screen Chinese medicinal herb extracts (CMHEs) with the aim of identifying a DDH inhibitor. Using this method, we found 49 among 796 CMHEs that inhibited DDH expression. We selected three potential extracts, which had the highest activity
\end{abstract}

Correspondence to: Dr Kuan-Chih Chow, Graduate Institute of Biomedical Sciences, National Chung Hsing University, 250 Kuo Kuang Road, Taichung 40227, Taiwan, R.O.C.

E-mail: kcchow@dragon.nchu.edu.tw

Abbreviations: AKR, aldo-keto reductase; ATAD3A, ATPase family AAA domain containing $3 \mathrm{~A} ; \mathrm{DDH}$, dihydrodiol dehydrogenase; DRP-1, dynamin-related protein-1; eEF2, eukaryotic elongation factor 2; ER, endoplasmic reticulum; ESCRT, endosomal sorting complex required for transport; HPLC, high-performance liquid chromatography; MAM, mitochondria-associated membrane; Mfn2, mitofusin 2; MMP, mitochondrial membrane potential; MOM, mitochondrial outer membrane; MVB, multivesicular body

Key words: dihydrodiol dehydrogenase, oncogene, drug detoxification, lipid flow, autophagy against DDH, for further fractionation using high-performance liquid chromatography. The active ingredient was identified by immunoblot analysis. The function of the active ingredient was characterized by cell function analysis. Our results revealed that the CMHE-purified compounds targeted DDH, inducing autophagy and reducing DNA repair, which in turn enhanced the cytotoxic effects of the anticancer drugs and irradiation.

\section{Introduction}

In a previous study, by combining differential display, reverse transcription polymerase chain reaction (RT-PCR) and DNA sequencing, we detected the overexpression of dihydrodiol dehydrogenase (DDH) in primary non-small cell lung cancer (NSCLC) specimens and lung cancer cell lines. We further found that DDH overexpression correlated with a higher frequency of tumor recurrence and distant metastasis (1). In retrospective studies, DDH overexpression was shown to correlate with poor prognosis, particularly in patients with late-stage disease $(1,2)$. By combining cDNA sequencing and two-dimensional gel electrophoresis, we identified that the $\mathrm{DDH}$ in the cancer cells belonged to aldo-keto reductase (AKR) family 1, member C1 (AKR1C1), and to a lesser extent to AKR family 1, member C2 (AKR1C2) (for the nomenclature of the respective enzymes, please refer to http://www.med.upenn. edu/akr/) (3). In vitro, the detection of DDH overexpression in ethacrynic acid-induced drug-resistant colon cancer cells and daunorubicin-resistant stomach cancer cells suggested that the involvement of DDH in the resistance of cancer cells to drugs may be a general phenomenon $(4,5)$. The fundamental nature of DDH to catabolize xenobiotic compounds indicates that the enzyme may deactivate anticancer drugs with similar polycyclic structures $(1,6,7)$.

However, using cDNA microarray to investigate genes which are overexpressed in cisplatin-resistant ovarian cancer 
cells, we also identified DDH. Subsequent studies of ectopic DDH expression confirmed our findings that DDH plays a role in the resistance of cancer cells to cisplatin (8). The evidently different chemical configurations and the evidently diverse resistance mechanisms between daunorubicin and cisplatin, which are respectively associated with cell membrane damage, inhibition of DNA topoisomerase II $\alpha$ activity and cross-linkage of double-stranded DNA $(9,10)$, nevertheless, raise the question as to how the two distinct anticancer agents converge on DDH expression. In particular in the latter case, in which cisplatin introduces DNA cross-linkage, as well as DNA strand breaks, indicates that the overexpression of DDH may also be involved in resistance to radiation (11). A previous study by Hung et al (12) demonstrated that cancer cells with higher expression levels of DDH, were indeed more resistant to irradiation. In fact, among the four subtypes of AKR1C, only DDH (AKR1C1) has been frequently detected in cancer cells, including bladder, esophageal, gastric, NSCLC, ovarian, prostate and uterine cervical cancer cells, suggesting that DDH acts as an oncogene in cancer progression (1,3,8,13-17).

Chen et al $(18,19)$ found that DDH overexpression suppressed the production of reactive oxygen species (ROS) and increased cisplatin resistance in ovarian and lung cancer cell lines. The silencing of DDH expression on the other hand, increased ROS levels and cisplatin sensitivity, supporting the data from other studies. Kruidering et al (20) demonstrated that cisplatin induced ROS production in the mitochondria by inhibiting glutathione reductase and activities of the respiratory chain. However, the increase in ROS production alone did not sufficiently kill the cancer cells, suggesting that other mechanisms, apart from the decrease in intracellular ROS levels by DDH may play a role in the inhibition of cell death. Nonetheless, no particular inhibitor against DDH has been identified to date. In this study, we therefore used DDH as a target enzyme in a live-cell enzyme-linked immunosorbent assay (http://www. piercenet.com) to screen a panel of Chinese medicinal herb extracts (CMHEs) in order to identify an inhibitor of DDH expression. The function of the potentially effective extracts, which were further fractionated by high-performance liquid chromatography (HPLC), was determined by immunoblot analysis and subsequent cell function analysis.

\section{Materials and methods}

Cell culture. Culture media and fetal calf serum (FCS) were from Gibco Laboratories (Grand Island, NY, USA). All other materials were of reagent grade and were obtained from Sigma (St. Louis, MO, USA), and Merck (Darmstadt, Germany). The lung cancer cells, H125, H226, H23, H838, H1437, H2009, H2087 and A549, the breast cancer cells, ZR-75-1, BT-20, MCF-1, MCF-7 and T47D, as well as the gastric cancer cells, AGS, KOTA-III, NUGC-1, NUGC-3 and SC-M1, were purchased from the American Type Culture Collection (Manassas, VA, USA), and were grown in monolayer in RPMI-1640 plus $10 \%$ FCS. All cultures were incubated at $37^{\circ} \mathrm{C}$ and all media were supplemented with $3 \mathrm{mM}$ glutamine, penicillin $(100 \mathrm{IU} / \mathrm{ml})$ and streptomycin $(100 \mu \mathrm{g} / \mathrm{ml})$.

Live-cell enzyme-linked immunosorbent assay (LCELISA) and colony forming assay for the determination of drug and radia- tion sensitivity. In a 96-well plate, 2,000 lung cancer cells were seeded into each well, and allowed to attach to the bottom of the well for at least $18 \mathrm{~h}$. Supernatants from 796 species of Chinese herbs, which were prepared at $0.5 \mathrm{~g} / \mathrm{ml}$ by collecting the supernatant from $0.5 \%$ ethanol extracts of the herbal powder. The extract was heated at $65^{\circ} \mathrm{C}$ for 30 min prior to collecting the supernatant by filtering through a $0.45 \mathrm{~nm}$ aseptic disc. The supernatant was respectively added to the wells at 1:200, 1:500 or 1:1250 dilutions, and incubated at $37^{\circ} \mathrm{C}$ for $72 \mathrm{~h}$ before fixing cells with $4 \%$ paraformaldehyde in phosphate-buffered saline (PBS) at room temperature for $15 \mathrm{~min}$. The cells were perforated with $0.1 \%$ Triton $\mathrm{X}-100$ for $5 \mathrm{~min}$ prior to the addition of antibodies to DDH to each well. The presence of DDH was detected by indirect immunocytochemistry.

Drug and radiation sensitivity were measured by the number of cells killed (21). Cells were seeded at 100, 1,000 and 10,000 cells $/ 6 \mathrm{~cm}$ plate $18 \mathrm{~h}$ prior to the drug or radiation challenge. The cells were treated with various doses of radiation or with various concentrations of anticancer drugs, such as cisplatin, for $2 \mathrm{~h}$ before removing drugs. The negative control groups included cells without radiation or cells treated with the same dilution of DMSO that was used as the solvent for the drug. The total number of survived cells was determined seven to ten days following drug challenge by crystal violet staining. The percentage survival of cells was quantified by comparing with the control group.

Immunoblot analysis. The procedure for immunoblot analysis was carried out as previously described $(1,3)$. Briefly, $5 \times 10^{6}$ cells were washed with PBS twice and lysed in loading buffer [50 mM Tris (pH 6.8), $150 \mathrm{mM} \mathrm{NaCl}, 1 \mathrm{mM}$ disodium EDTA, 5\% $\beta$-mercaptomethanol, $1 \mathrm{mM}$ phenylmethylsulfonyl fluoride, $10 \%$ glycerol, $1 \%$ SDS and $0.01 \%$ bromophenol blue]. Electrophoresis was carried out in a $10 \%$ polyacrylamide gel with $4.5 \%$ stacking gel. Following electrophoresis, the proteins were transferred oonto a nitrocellulose membrane. The membrane was then probed with specific antibodies. The signal was amplified by biotin-labeled goat anti-mouse IgG and peroxidase-conjugated streptavidin. Protein was visualized by exposing the membrane to an X-Omat film (Eastman Kodak, Rochester, NY, USA) with enhanced chemiluminescence reagent (Pierce, Rockford, IL, USA).

Electron microscopy. Electron microscopy was performed following a previously published protocol $(22,23)$. Briefly, the cells were fixed with $2.5 \%$ glutaraldehyde (EM grade; Sigma) in $100 \mathrm{mM}$ phosphate buffer (PB, pH 7.2) at $4^{\circ} \mathrm{C}$ for $18 \mathrm{~h}$. The cells were rinsed with $\mathrm{PB}$ prior to post-fixation with $1 \%$ osmium tetroxide. After removal of the fixative with distilled water, the cells were suspended in $2 \%$ molten agar $\left(42^{\circ} \mathrm{C}\right)$, and the agar was then allowed to solidify. The trimmed agar blocks were dehydrated in a serial dilution of ethanol (absolute alcohol) for $15 \mathrm{~min}$ each, and then infiltrated with $100 \%$ ethanol/LR white (1:1) mixture for $18 \mathrm{~h}$. The blocks were changed to pure LR white (Agar Scientific Ltd., Essex, UK) and infiltration was continued at $4^{\circ} \mathrm{C}$ for $24 \mathrm{~h}$, prior to transfer to a capsule filled with LR white, and were then polymerized and solidified at $60^{\circ} \mathrm{C}$ for $48 \mathrm{~h}$. The trimmed resin blocks were cut using an ultramicrotome (Leica Ultracut R; Leica Microsystems $\mathrm{GmbH}$, Vienna, Austria), and the thin sections 
were transferred onto 200 mesh copper grids. The specimens were stained with $2 \%$ uranyl acetate for $30 \mathrm{~min}$, and $2.66 \%$ lead citrate $(\mathrm{pH} 12.0)$ for 10 min prior to observation using an electron microscope (JEM-1400; Jeol USA, Inc., Peabody, MA, USA) at $100-120 \mathrm{kV}$.

Immunofluorescence staining and immunofluorescence microscopy. For immunofluorescence microscopy, the cellular uptake of MitoTracker ${ }^{\circledR}$ Green FM (Molecular Probes, Inc., Eugene, OR, USA) was used to label the mitochondria $(22,24)$. The cells were then fixed with $4 \%$ formaldehyde at room temperature for $15 \mathrm{~min}$. After washing with PBS, the cells were incubated with the primary antibodies to DDH as previously described (1) or ceramide (Enzo Life Sciences, Inc., Farmingdale, NY, USA) for $90 \mathrm{~min}$ and then washed with PBS. The secondary antibodies used were rhodamine (TRITC)-conjugated rabbit anti-mouse IgG (Jackson Laboratories, West Grove, PA, USA). The nuclei were stained with 4',6-diamidino-2-phenylindole (DAPI). The slide was examined and images were captured using an immunofluorescence microscope with an UIS2 optical system (Olympus BX51; Olympus Corp., Tokyo, Japan). The images were processed using Olympus DP2-BSW image capture software (Olympus Corp.) and Adobe Photoshop 7.01 software (Adobe Systems, Inc., San Jose, CA, USA).

The cells, which were transfected with organelle-specific plasmids, human phosphatidylserine synthase 1 [FH-hPSS1, a marker enzyme for the endoplasmic reticulum (ER), mitochondria-associated membrane (MAM) and microsomes] or galactosyltransferase-conjugated green fluorescent protein (GT-GFP, as a marker for the Golgi apparatus) for $24 \mathrm{~h}$, were seeded onto slides. After $48 \mathrm{~h}$, the cells were fixed with $4 \%$ paraformaldehyde for $15 \mathrm{~min}$ at room temperature and analyzed by fluorescence microscopy. For the ceramide- and FH-hPSS1-expressing cells, the cells were permeabilized with $0.1 \%$ Triton $\mathrm{X}-100$ for $15 \mathrm{~min}$ at room temperature, and subsequently stained with mouse anti-HA tag (Santa Cruz Biotechnology, Inc., Santa Cruz, CA, USA), and fluoresceinconjugated anti-mouse IgG antibodies (Invitrogen, Carlsbad, CA, USA).

Statistical analysis. The two-way ANOVA test was performed using GraphPad Prism 5 statistical software (GraphPad Software. Inc., San Diego, CA, USA). A p-value $<0.05$ was considered to indicate a statistically significant difference.

\section{Results}

Selection of cancer cell lines with high levels of DDH expression. Among the eight lung cancer cell lines screened by immunoblot analysis, DDH was highly expressed in the H838, H1437 and A549 cells, and weakly expressed in the H226 and H2087 cells. DDH was not detected in the H23, H125 and H2009 cells (Fig. 1A). Among the five breast cancer cell lines, DDH was detected in the BT-20, MCF-1 and MCF-7 cells (Fig. 1B), and among the five gastric cancer cell lines, DDH was detected in the KATO-III, NUGC-1 and SC-M1 cells (Fig. 1C). We therefore selected to use the H838, BT-20 and KATO-III cells in the screening of the 796 CMHEs, which may contain vital ingredients that could suppress DDH protein expression, but not immediately kill the cells.
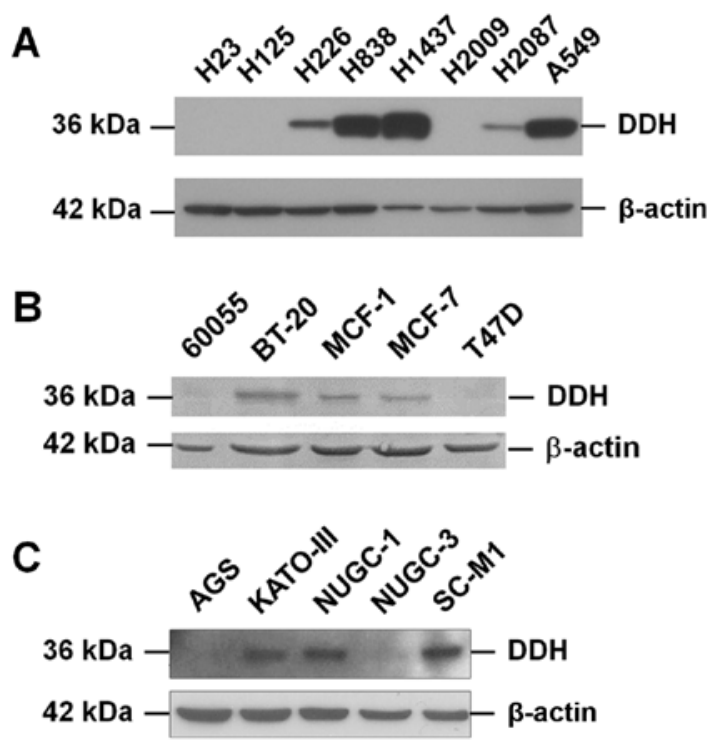

Figure 1. Selection of cancer cell lines with a high expression of dihydrodiol dehydrogenase (DDH). (A) Among the eight lung cancer cell lines examined by immunoblot analysis, DDH was highly expressed in the H838, $\mathrm{H} 1437$ and A549 cells, and weak expressed in the H226 and H2087 cells. DDH was not detected in the H23, H125 and H2009 cells. (B) Among the five breast cancer cell lines, DDH was detected in the BT-20, MCF-1 and MCF-7 cells. (C) Among the five gastric cancer cell lines, DDH was detected in the KATO-III, NUGC-1 and SC-M1 cells. The H838, BT-20 and KATO-III cells were thus selected for use in screening Chinese medicinal herb extracts (CMHEs), which may contain ingredients that inhibit DDH protein expression, but not immediately kill the cells. Our aim was to find CMHE ingredients, which can inhibit DDH activity, and those that are specific for cancer progression. The addition of these ingredients could then increase cytosensitivity to anticancer drugs and radiation.

Effects of CMHE on the suppression of DDH protein expression as determined by LCELISA and immunoblot analysis. Using LCELISA to screen the 796 CMHEs, we identified 49 extracts that suppressed DDH protein expression (Fig. 2A). The results were confirmed by immunoblot analysis (Fig. 2B). Although some CMHEs suppressed DDH protein expression and induced cell death, most of the CMHEs induced DDH expression and enhanced cell growth.

Isolation and characterization of pure compounds in CMHEs which suppress DDH expression. As shown in Fig. 2A, as CMHE-12 [from Nothapodytes foetida (Wight) Sleumer] and CMHE-15 (from Koelreuteria henryi Dummer) had a greater suppressive effect on DDH expression, we thus fractionated the pure compounds using HPLC from these two extracts and characterized the anti-DDH activities. The major compound isolated from CMHE-12, which markedly inhibited DDH expression, turned out to be camptothecin (25), which induced the cleavage of poly(ADP-ribose) polymerase (PARP) (Fig. 3A) and apoptosis. From CMHE-15, among the final six fractions, four compounds were identified by nuclear magnetic resonance (NMR) (Fig. 3B); however, only two compounds contained effective ingredients (Fig. 3C). Similar to CMHE-12, compound G (apigenin-4'-O- $\beta$ glucopyranoside) induced PARP cleavage and apoptosis, but did not markedly inhibit DDH expression, nor synergistically enhance the cytotoxicity of cisplatin, adriamycin, vincristine, etoposide or radiation (data not shown). Compound B, which 
A

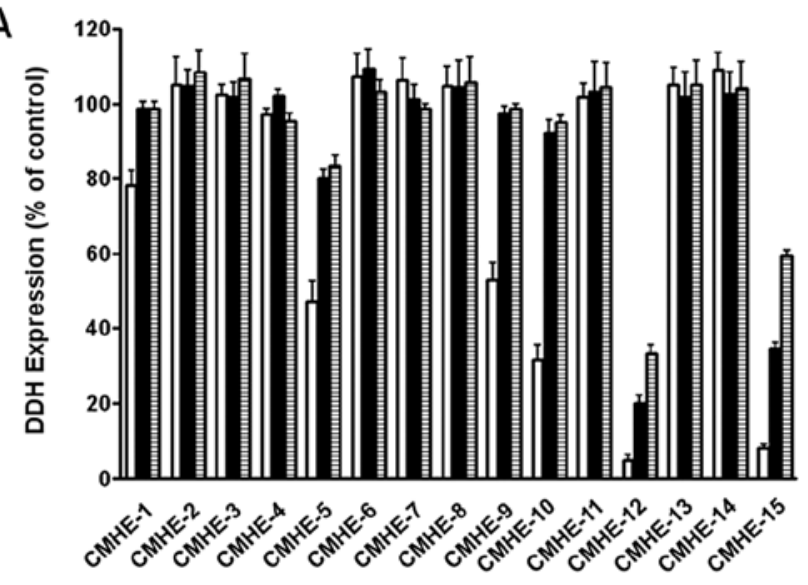

B

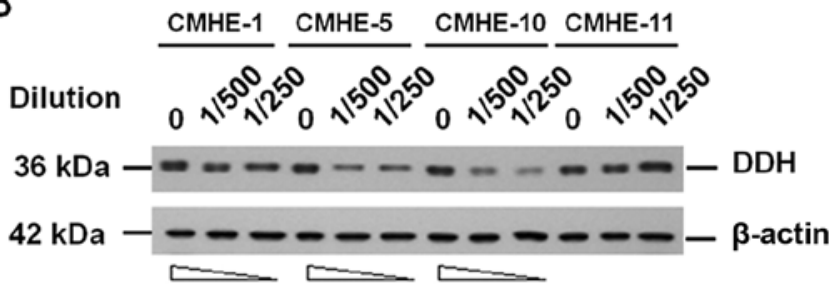

Figure 2. Use of LCELISA and immunoblot analysis to determine the effects of Chinese medicinal herb extracts (CMHEs) on the inhibition of dihydrodiol dehydrogenase (DDH) protein expression. (A) Using LCELISA, we screened 796 CMHEs, and 49 were found to inhibit DDH protein expression. Results are shown for the first 15 samples. Bars indicate: $\square$, H838; $\mathbf{m}$, BT-20; $\equiv$, KATO-III. (B) The results of LCELISA were confirmed by immunoblot analysis. CMHE-5 and CMHE-10 markedly reduced DDH protein expression.

intermediately suppressed DDH protein expression, however, did not extensively induce PARP cleavage, but synergistically enhanced the cytotoxic effects of the anticancer drugs and irradiation. Using NMR, compound B was characterized as kaempferol-3-O-glucoside (Fig. 3C), also known as astragalin, an abundant ingredient in the Chinese medicinal herb, Astragalus membranaceus (Huang-qi) (26). Although by conformation, compound I (austrobailignan-1) resembled epipodophyllotoxins, it did not induce PARP cleavage.

Cytological effects of astragalin. Astragalin, which intermediately inhibited DDH protein expression, did not induce the cleavage of PARP (Fig. 4A), but reduced the levels of ATPase family AAA domain containing 3A (ATAD3A) and dynaminrelated protein-1 (DRP-1), and induced the conversion of LC-3-I to LC3-II (Fig. 4B). Although astragalin increased the levels of pro-caspase-3, pro-caspase-8 and Bax (Fig. 4C), no evident activation of pro-caspase- 3 or pro-caspase- 8 cleavage was observed. On the other hand, astragalin reduced the mitochondrial membrane potential (MMP) (Fig. 4D), suggesting that astragalin induces cell death through mechanisms other than the apoptotic pathway. In fact, the addition of astragalin increased the intracellular numbers of autophagic vacuoles (Fig. 4E), indicating that astragalin indeed induced autophagy (Fig. 4F). In this way, the addition of astragalin enhanced the cytosensitivity of the cancer cells to the anticancer drugs and radiation (Fig. 4G).
A CMHE-12

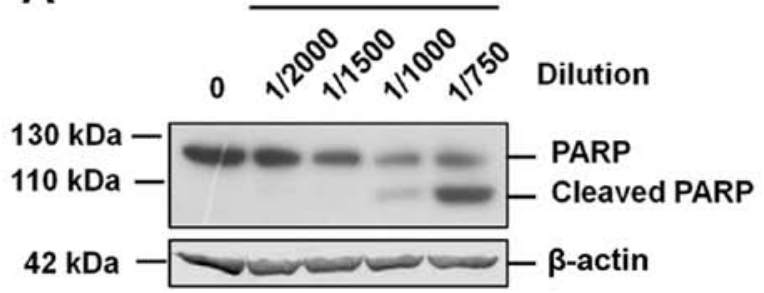

B
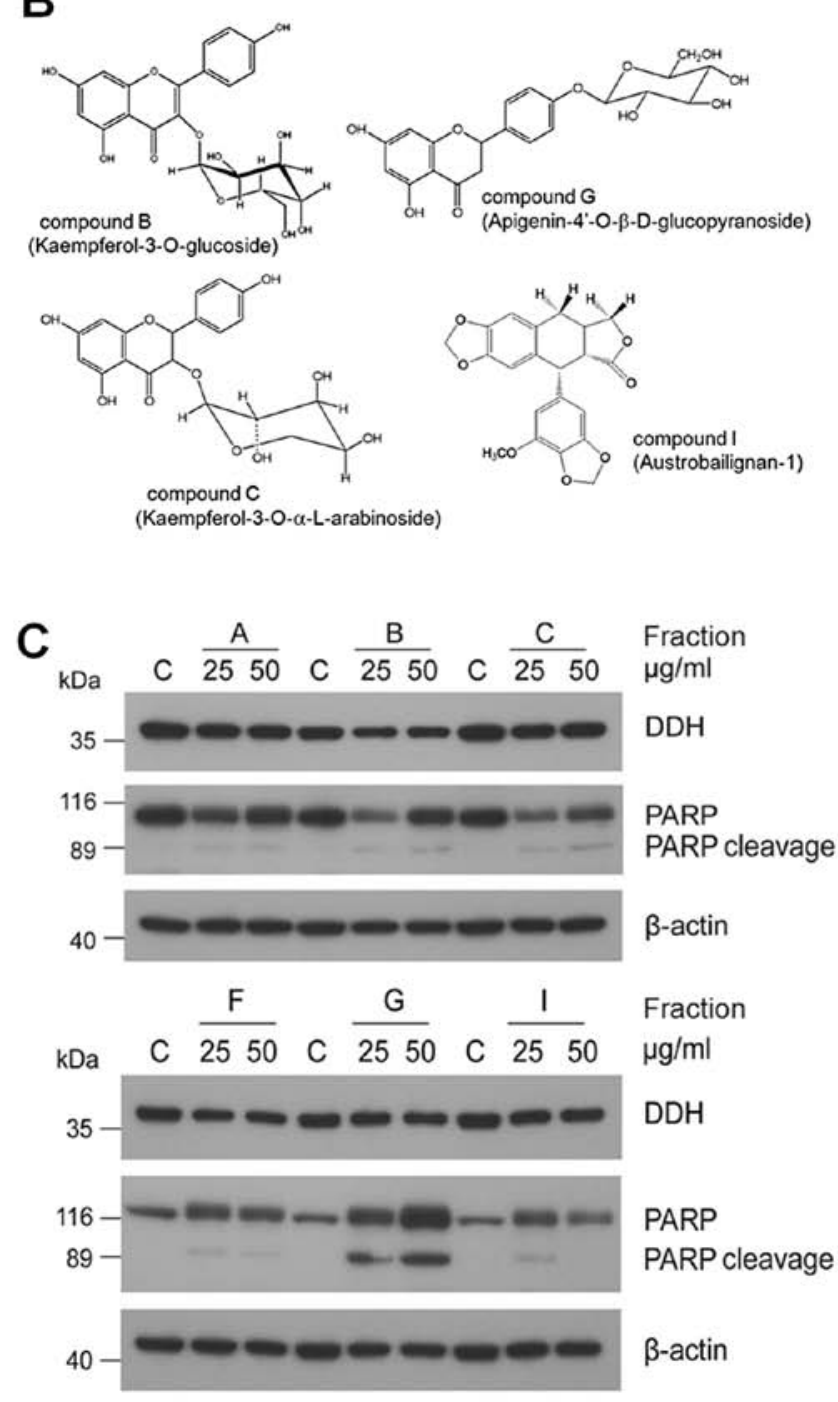

Figure 3. Isolation and characterization of pure compounds in the Chinese medicinal herb extract (CMHE) which markedly suppressed dihydrodiol dehydrogenase (DDH) protein expression. As CMHE-12 and -15 had the strongest inhibitory effect on DDH protein expression, the active ingredients in these two plant extracts were fractionated using high-performance liquid chromatography (HPLC), and characterized by LCELISA as well as immunoblot analysis. (A) From CMHE-12, the major ingredient isolated was identified to be camptothecin, which not only reduced $\mathrm{DDH}$ protein expression, but also induced the cleavage of poly(ADP-ribose) polymerase (PARP), a marker of apoptosis. (B) Among the six final fractions of CMHE-15, five contained ingredients which effectively inhibited DDH expression. (C) The identity of four compounds was determined by nuclear magnetic resonance. Compound C (kaempferol-3-O-a-arabinoside) and compound I (austrobailignan) did not effectively inhibit DDH or induce apoptosis. Although compound G (apigenin-4'-O-b-D-glucopyranoside) markedly induced the cleavage of PARP, it did not effectively inhibit DDH expression nor did it synergistically enhance the effects of the anticancer drugs or radiation (data not shown). As compound B (kaempferol-3-O-glucoside, astragalin), intermediately suppressed DDH protein expression, but did not extensively induce PARP cleavage, its cytological effects were further examined. 
A

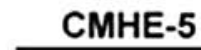

- $1^{\circ} 2^{\circ} 3^{\circ} \mu \mathrm{g} / \mathrm{ml}$

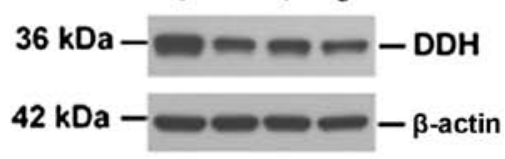

B

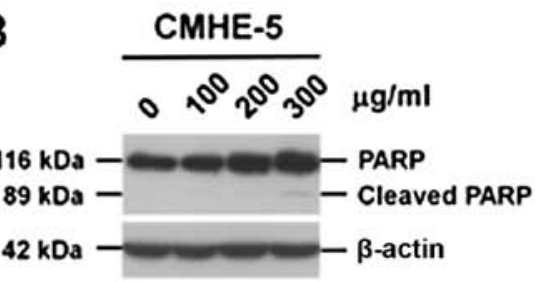

\section{CMHE-5

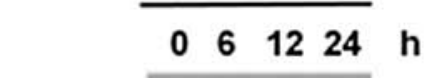

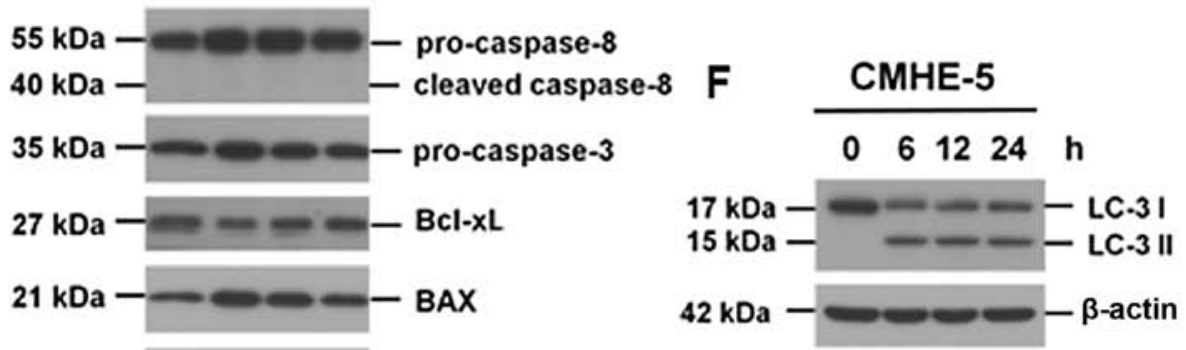

42 kDa $-1-\beta$-actin
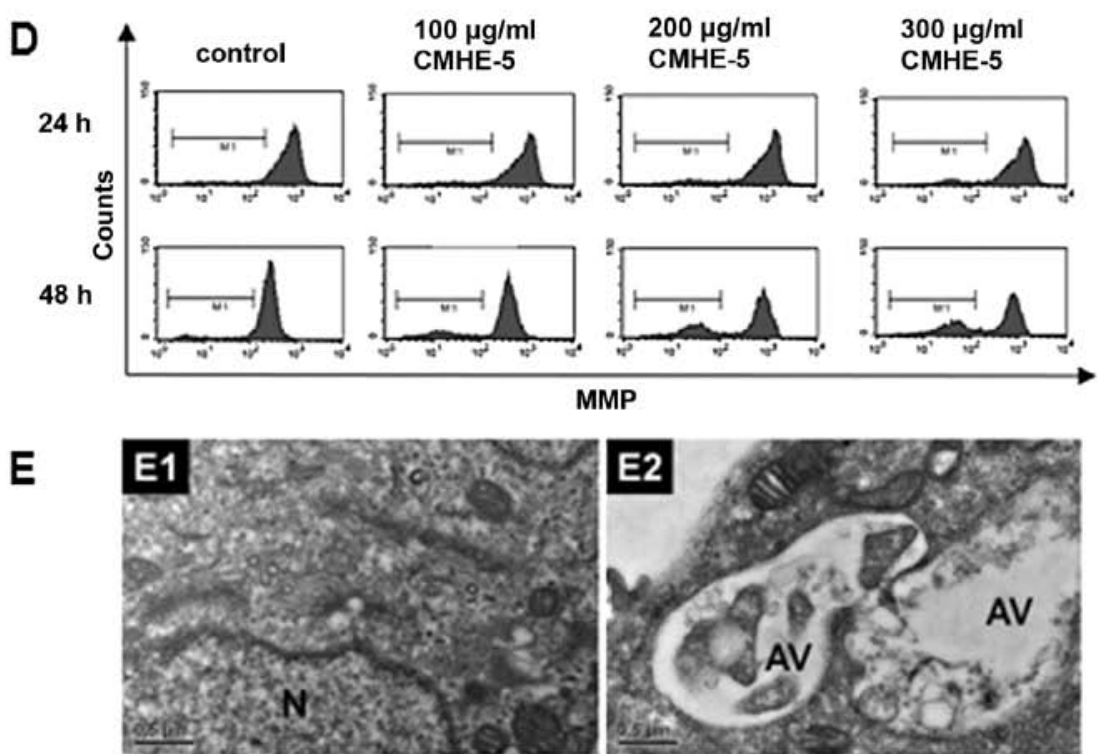

G

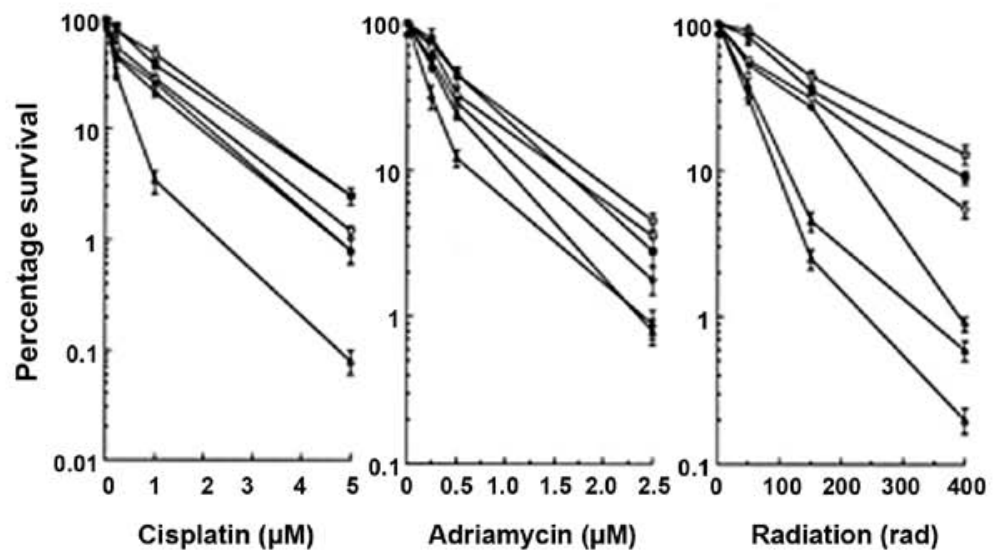

Figure 4. Cytological changes induced by astragalin. (A) Astragalin inhibited dihydrodiol dehydrogenase (DDH) expression in a time-dependent manner. However, it did not induce the extensive cleavage of poly(ADP-ribose) polymerase (PARP). (B) Astragalin also suppressed the expressions of ATPase family AAA domain containing 3A (ATAD3A) and dynamin-related protein-1 (DRP-1), and increased the conversion of LC-3-I to LC3-II, suggesting that astragalin may be able to induce autophagy. (C) Astragalin markedly increased the protein levels of pro-caspase-3, pro-caspase- 8 and Bax, but it did not induce the cleavage of pro-caspase-3 or -8. (D) Treatment with astragalin reduced mitochondrial membrane potential (MMP), supporting the previous observations that astragalin may be able to induce cell death, other than apoptosis. (E) Astragalin increased intracellular numbers of autophagic vacuoles as determined by electron microscopy. (F) Astragalin increased the conversion of LC3-I to LC-3II, an authentic autophagic marker as determined by immunoblot analysis. (G) Astragalin increased the cytosensitivity of cancer cells to the anticancer drugs, such as cisplatin and adriamycin, as well as radiation. 


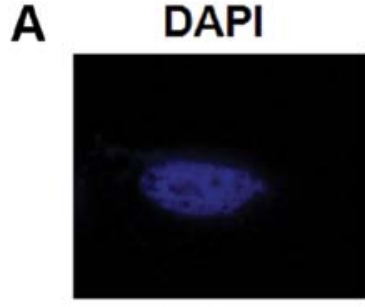

DAPI

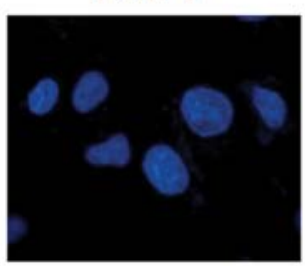

DAPI

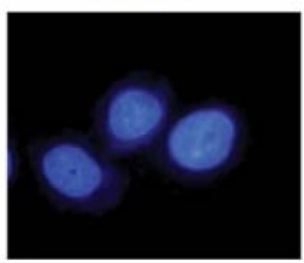

B

Control

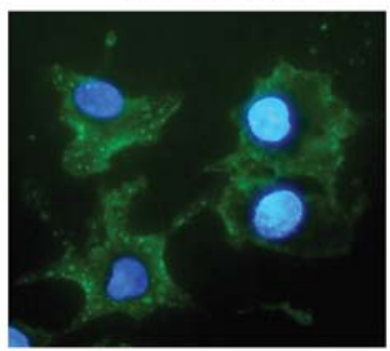

PSS1-GFP

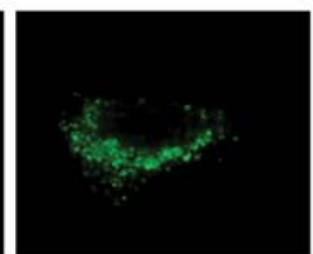

GT-GFP

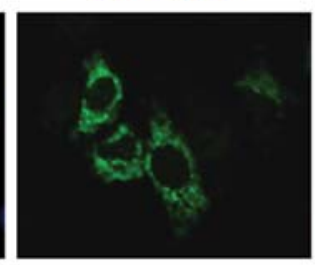

MitoTracker

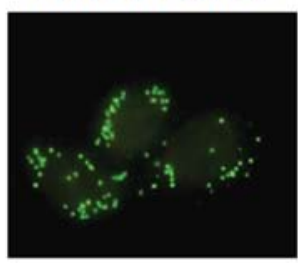

DDH

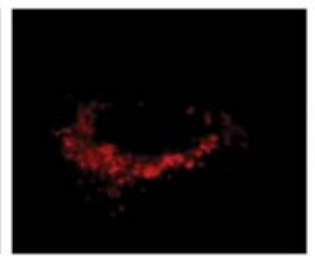

DDH

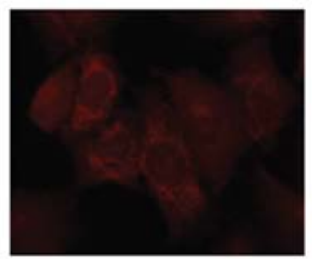

DDH

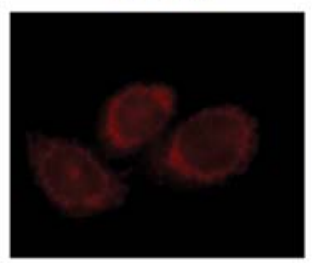

Merge

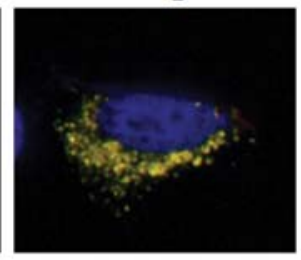

Merge

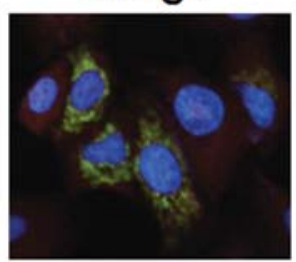

Merge

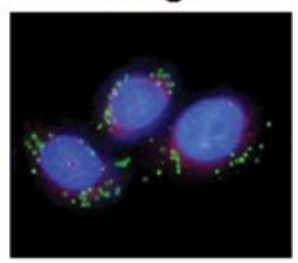

Astragalin
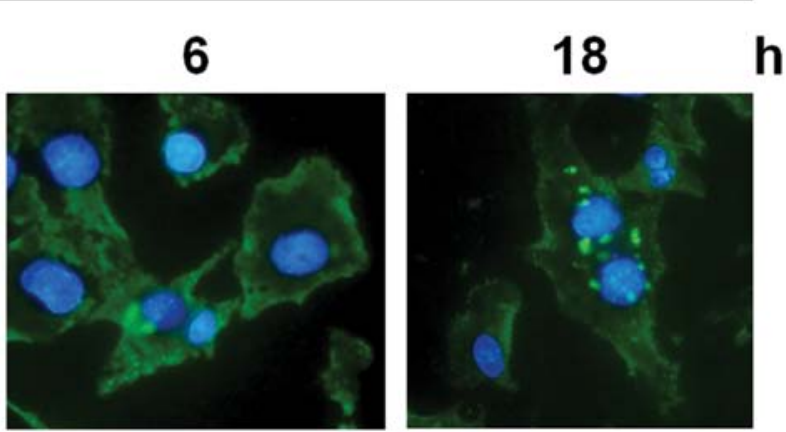

Figure 5. Intracellular location of dihydrodiol dehydrogenase (DDH), and the effects of the inhibition of DDH expression on intracellular ceramide distribution, as well as the induction of autophagy. (A) Using immunofluorescence microscopy, DDH was found to be localized to the endoplasmic reticulum (ER), mitochondria-associated membrane (MAM), microsomes and Golgi apparatus, but not to the mitochondria. (B) In the control group, ceramide was localized on the intracellular vesicular structures and plasma membrane as determined by an immunofluorescence microscopy (left panel). Treatment with compound B inhibited DDH expression for $6 \mathrm{~h}$; on the other hand, it reduced ceramide levels on the intracellular vesicular structures and plasma membrane. Ceramide was accumulated on the Golgi-like structures (center panel). At $18 \mathrm{~h}$ post-treatment with compound B, ceramide was mostly concentrated on autophagy-like vesicles (right panel). Ceramide was detected by an immunofluorescence method.

Astragalin reduces ceramide distribution to the plasma membrane, but increases intracellular autophagic vesicles. As shown in Fig. 5A, using immunofluorescence microscopy, DDH was localized to the ER, MAM, microsomes (Fig. 5A, top panel) and Golgi apparatus (Fig. 5A, middle panel), but not on the mitochondria (Fig. 5A, lower panel). The addition of astragalin, however, did not affect DDH localization to the organelles (data not shown).

On the other hand, astragalin altered the intracellular distribution of ceramide. Ceramide (27), which is normally localized on the intracellular vesicular structures and plasma membrane (Fig. 5B, left panel), was accumulated on MAM- or Golgi-like structures (Fig. 5B, center panel) $6 \mathrm{~h}$ following treatment with astragalin. At $18 \mathrm{~h}$ post-astragalin treatment, ceramide was amassed on the autophagic vesicles (Fig. 5B, right panel), suggesting that the inhibition of DDH expression disrupted the intracellular transportation/distribution of ceramide.

\section{Discussion}

Our data demonstrate that extracts from Taiwan-endemic roadside trees, Koelreuteria henryi Dummer, contained at least two ingredients, which respectively induced apoptosis and autophagy, thus enhancing the cytotoxic effects of the anticancer drugs and irradiation. Using HPLC and NMR, one of the active compounds isolated from the plant extracts was kaempferol-3-O-glucoside (or astragalin), a major ingredient in the traditional Chinese medicinal herb, Astragalus membranaceus 
(Huang-qi). Unlike herbal Astragalus membranaceus though, Koelreuteria henryi Dummer is deciduous and the contents of astragalin and apigenin-4'-O- $\beta$-glucopyranoside fluctuate as the seasons change. Apigenin-4'-O- $\beta$-glucopyranoside extensively induced caspase-dependent apoptosis. However, astragalin only induced autophagy possibly by interrupting the protein and lipid flow among the organelles, and this concept was clearly supported by the immunofluorescence microscopic observations that astragalin markedly altered the intracellular distribution of ceramide, which was accumulated on the MAM- and Golgi-like finite configurations.

The identification of DDH on the microsomes, Golgi apparatus, ER and MAM, but not on the mitochondria consolidated our theory, which supports not only previous studies, in which $\mathrm{DDH}$ has been shown to deactivate doxorubicin, an anticancer drug with a polycyclic hydrophobic side-chain $(4,5)$, but also evidence that DDH catabolizes hydrophobic xenobiotic compounds, in particular polycyclic aromatic hydrocarbons (PAHs), which are more likely to be associated with lipid membranes (4-7). Furthermore, the results corresponded well with the observations that DDH had the worst effect on tumor progression when the enzyme was concurrently overexpressed with microsomal epoxide hydrolase (28). As reported previously, Chen et al $(18,19)$ found that higher DDH levels correlated with the reduction of mitochondrial ROS concentrations. The silencing of DDH, on the other hand, increased ROS levels. Moreover, Nie et al (29) showed that elevated intracellular ROS levels increased the oxidation of cys ${ }^{62}$ in Bax, which then induced the membrane perforation of the mitochondria and triggered apoptosis (30-32). However, by demonstrating that DDH was not located on the mitochondria and that changes in MMP did not occur after $24 \mathrm{~h}$ of astragalin treatment, our data suggest that the effects of DDH on ROS production may be indirect and that the cell death mediated by astragalin may be autophagic.

Recently, we found a novel import passage of the mitochondria, which originates from the ER and via transport vesicles $(22,24)$. This pathway requires DRP-1 (33), ATAD3A (24) and mitofusin-2 (Mfn2) $(22,24)$. The knockdown of the ATAD3A gene markedly reduced the protein levels of DDH and global DNA repair, but increased autophagy $(22,24)$. The silencing of DRP-1 expression induced a comparable autophagic response (33). Of note, apart from DDH, astragalin inhibited the expression of DRP-1 and ATAD3A. A poor supply of cytoplasmic DRP-1, on the other hand, increases mitochondrial fusion and the bulging of MAM, a specific region of the ER, part of which tethers the ER and mitochondria together by $\mathrm{Mfn} 2$ on both organelles to coordinate $\mathrm{Ca}^{2+}$ flow $(34,35)$, and from which the newly synthesized proteins, phospholipids and sphingolipids are respectively via transport vesicles imported to the mitochondria $(22,24)$ or exported to the Golgi and plasma membrane (3).

In fact, the cellular location of ceramide, which is synthesized specifically on the inner leaflet of the ER and is finally located on the outer leaflet of the plasma membrane following export through the Golgi apparatus and transport vesicles (27), has casted an intriguing question as to its biological function. In particular, the exogenously added ceramide induced autophagy (36). It is worth noting that ceramide is a hydrophobic compound, and is associated with membrane structure or carrier proteins. Under normal conditions, it is rapidly converted to sphingosine by cerases or sphingomyelin by sphingomyelin synthase, preventing the accidental induction of differentiation or cell death $(27,36)$.

Previous studies have demonstrated that the silencing of DDH expression reduces the levels of vacuolar protein sorting (Vps)4 (37), an essential ATPase for the movement of multivesicular body (MVB) in the endosomal sorting complex required for transport (ESCRT), and increases those of autophagy-related gene (Atg) 16L2 (38). Of note, ATAD3A shares some homology with Vps4 in the ATPase domain and with Atg16L2 in coiled-coil domains. The silencing of Vps4 induces autophagy as well. Although these results are yet to be verified, these data, together with evidence of DDH collaborating with epoxide hydrolase to fully detoxify hydrophobic PAHs into catechol (4-7,39-41), suggest that these enzymes are located in the near vicinity of membranous structures to guard the proper intracellular trafficking of proteins and lipids. Since the type of epoxide hydrolase, which has been commonly detected in cancer cells, is microsomal epoxide hydrolase (28), it is possible that DDH is also located in the immediate vicinity of these subcellular structures, which are originally from the ER and MAM. Our results demonstrated that DDH was not only located on the ER and MAM, but also on the Golgi apparatus, indicating that it may have a biological role apart from PAH detoxification. The interruption of these processes, i.e., by RNAi or small molecules derived from CMHEs, would certainly affect the enzyme activity involved in lipid flow and thus lead to the accumulation of toxic lipids in the organelles and interorganelle transport vesicles, inducing aberrant autophagy and inhibiting tumor progression.

In conclusion, our results revealed that DDH can indeed be used as a target enzyme for screening CMHEs, which contained potential ingredients able to inhibit DDH expression. The inhibition of DDH function reduced the expression of anti-apoptotic proteins, such as Bcl-xL, but increased the expression of pro-apoptotic proteins, such as Bax, and caspase precursors, including pro-caspase- 3 and -8 . However, the suppression of DDH alone did not induce apoptosis, but induced autophagy, thus enhancing the sensitivity to anticancer drugs and radiation. This weakening activity was specific for cancer cells, which not only highly expressed DDH, but also DRP-1, ATAD3A and eukaryotic elongation factor 2 (eEF2) $(24,33,42,43)$, a condition that was not detected in non-transformed cells. Moreover, some of the purified prospective ingredients concurrently inhibited both DDH and ATAD3A, and the latter is an ATPase which is closely associated with the intracellular transport of proteins, phospholipids and sphingolipids. The silencing of ATAD3A induced massive autophagy and enhanced cytosensitivity to anticancer drugs and radiation, suggesting that these prospective ingredients may not only selectively target $\mathrm{DDH}$, but also a spectrum of cancer progression-related genes, which are involved in the interorganelle transportation of essential proteins and lipids.

\section{Acknowledgements}

The present study was supported by grants from the Department of Health, Executive Yuan, Taipei, Taiwan to the China Medical University Hospital, the Cancer Research of Excellence program (DOH102-TD-C-111-005), 
Taichung, Taiwan, and the National Science Council (NSC, 101-2320-B-005-002), Taipei, Taiwan.

\section{References}

1. Hsu NY, Ho HC, Chow KC, et al: Overexpression of dihydrodiol dehydrogenase as a prognostic marker of non-small cell lung cancer. Cancer Res 61: 2727-2731, 2001.

2. Chen CY, Hsu CP, Hsu NY, Shih CS, Lin TY, and Chow KC: Expression of dihydrodiol dehydrogenase in the resected stage I non-small cell lung cancer. Oncol Rep 9: 515-519, 2002.

3. Huang KH, Chiou SH, Chow KC, et al: Overexpression of aldo-keto reductase $1 \mathrm{C} 2$ is associated with disease progression in patients with prostatic cancer. Histopathology 57: 384-394, 2010.

4. Shen H, Kauvar L and Tew KD: Importance of glutathione and associated enzymes in drug response. Oncol Res 9: 295-302, 1997.

5. Ax W, Soldan M, Koch L and Maser E: Development of daunorubicin resistance in tumour cells by induction of carbonyl reduction. Biochem Pharmacol 59: 293-300, 2000.

6. Shou M, Harvey RG and Penning TM: Contribution of dihydrodiol dehydrogenase to the metabolism of (+/-)-trans7,8-dihydroxy-7,8-dihydrobenzo[a]pyrene in fortified rat liver subcellular fractions. Carcinogenesis 13: 1575-1582, 1992.

7. Flowers-Geary L, Harvey RG and Penning TM: Cytotoxicity of polycyclic aromatic hydrocarbon o-quinones in rat and human hepatoma cells. Chem Res Toxicol 6: 252-260, 1993

8. Deng HB, Parekh HK, Chow KC and Simpkins H: Increased expression of dihydrodiol dehydrogenase induces resistance to cisplatin in human ovarian carcinoma cells. J Biol Chem 277: 15035-15043, 2002.

9. Chow KC, MacDonald TL and Ross WE: DNA binding by epipodophyllotoxins and $\mathrm{N}$-acyl anthracyclines: implications for mechanism of topoisomerase II inhibition. Mol Pharmacol 34 467-473, 1988

10. Hill BT, Shellard SA, Hosking LK, Fichtinger-Schepman AM and Bedford P: Enhanced DNA repair and tolerance of DNA damage associated with resistance to cis-diammine-dichloroplatinum (II) after in vitro exposure of a human teratoma cell line to fractionated X-irradiation. Int J Radiat Oncol Biol Phys 19: $75-83,1990$.

11. Sorenson CM and Eastman A: Mechanism of cisdiamminedichloroplatinum(II)-induced cytotoxicity: role of G2 arrest and DNA double-strand breaks. Cancer Res 48: 4484-4488, 1988.

12. Hung JJ, Chow KC, Wang HW and Wang LS: Expression of dihydrodiol dehydrogenase and resistance to chemotherapy and radiotherapy in adenocarcinoma cells of lung. Anticancer Res 26: 2949-2955, 2006.

13. Wang LS, Chow KC, Wu YC, Lin TY and Li WY: Inverse expression of dihydrodiol dehydrogenase and glutathione-Stransferase in patients with esophageal squamous cell carcinoma. Int J Cancer 111: 246-251, 2004.

14. Chow KC, Lu MP and Wu MT: Expression of dihydrodiol dehydrogenase plays important roles in apoptosis- and drugresistance of A431 squamous cell carcinoma. J Dermatol Sci 41 205-212, 2006

15. Ueda M, Hung YC, Chen JT, et al: Infection of human papillomavirus and overexpression of dihydrodiol dehydrogenase in uterine cervical cancer. Gynecol Oncol 102: 173-181, 2006.

16. Tai HL, Lin TS, Huang HH, et al: Overexpression of aldo-keto reductase $1 \mathrm{C} 2$ as a high-risk factor in bladder cancer. Oncol Rep 17: 305-311, 2007.

17. Chang HC, Chen YL, Chan CP, et al: Overexpression of dihydrodiol dehydrogenase as a prognostic marker in resected gastric cancer patients. Dig Dis Sci 54: 342-347, 2009.

18. Chen J, Adikari M, Pallai R, Parekh HK and Simpkins H: Dihydrodiol dehydrogenases regulate the generation of reactive oxygen species and the development of cisplatin resistance in human ovarian carcinoma cells. Cancer Chemother Pharmacol 61: 979-987, 2008.

19. Chen J, Emara N, Solomides C, Parekh H and Simpkins H: Resistance to platinum-based chemotherapy in lung cancer cell lines. Cancer Chemother Pharmacol 66: 1103-1111, 2010.

20. Kruidering M, Van de Water B, de Heer E, Mulder GJ and Nagelkerke JF: Cisplatin-induced nephrotoxicity in porcine proximal tubular cells: mitochondrial dysfunction by inhibition of complexes I to IV of the respiratory chain. J Pharmacol Exp Ther 280: 638-649, 1997.
21. Chow KC, King CK and Ross WE: Abrogation of etoposidemediated cytotoxicity by cycloheximide. Biochem Pharmaco 137: 1117-1122, 1988.

22. Chiang SF, Huang CY, Lin TY, Chiou SH and Chow KC: An alternative import pathway of AIF to the mitochondria. Int J Mol Med 29: 365-372, 2012.

23. Yoon Y, Pitts KR, Dahan S and McNiven MA: A novel dynaminlike protein associates with cytoplasmic vesicles and tubules of the endoplasmic reticulum in mammalian cells. J Cell Biol 140: 779-793, 1998.

24. Fang HY, Chang CL, Hsu SH, et al: The ATPase family, AAA domain containing $3 \mathrm{~A}$ is a novel anti-apoptotic factor in lung adenocarcinoma cells. J Cell Sci 123: 1171-1180, 2010.

25. Aiyama R, Nagai H, Nokata K, Shinohara C and Sawada S: A camptothecin derivative from Nothapodytes foetida. Phytochemistry 27: 3663-3664, 1988.

26. Kwon HJ and Park YD: Determination of astragalin and astragaloside content in Radix Astragali using high-performance liquid chromatography coupled with pulsed amperometric detection. J Chromatogr A 1232: 212-217, 2012.

27. Zheng W, Kollmeyer J, Symolon H, et al: Ceramides and other bioactive sphingolipid backbones in health and disease: lipidomic analysis, metabolism and roles in membrane structure, dynamics, signaling and autophagy. Biochim Biophys Acta 1758: 1864-1884, 2006.

28. Lin TS, Huang HH, Fan YH, Chiou SH and Chow KC: Genetic polymorphism and gene expression of microsomal epoxide hydrolase in non-small cell lung cancer. Oncol Rep 17: 565-572, 2007.

29. Nie C, Tian C, Zhao L, Petit PX, Mehrpour M and Chen Q: Cysteine 62 of $\mathrm{Bax}$ is critical for its conformational activation and its proapoptotic activity in response to $\mathrm{H}_{2} \mathrm{O}_{2}$-induced apoptosis. $\mathrm{J}$ Biol Chem 283: 15359-15369, 2008.

30. Szabò I, Soddemann M, Leanza L, Zoratti M and Gulbins E: Single-point mutations of a lysine residue change function of $\mathrm{Bax}$ and Bcl-xL expressed in Bax- and Bak-less mouse embryonic fibroblasts: novel insights into the molecular mechanisms of Bax-induced apoptosis. Cell Death Differ 18: 427-438, 2011.

31. Griparic L, van der Wel NN, Orozco IJ, Peters PJ and van der Bliek AM: Loss of the intermembrane space protein Mgm1/OPA1 induces swelling and localized constrictions along the lengths of mitochondria. J Biol Chem 279: 18792-18798, 2004.

32. Fang HY, Chen CY, Chiou SH, et al: Overexpression of OPA1 protein increases cisplatin resistance via inactivation of caspasedependent apoptosis in lung adenocarcinoma cells. Hum Pathol 43: 105-114, 2012.

33. Chiang YY, Chen SL, Hsiao YT, et al: Nuclear expression of dynamin-related protein 1 in lung adenocarcinomas. Mod Pathol 22: 1139-1150, 2009

34. de Brito OM and Scorrano L: Mitofusin 2 tethers endoplasmic reticulum to mitochondria. Nature 456: 605-610, 2008.

35. Merkwirth $C$ and Langer T: Mitofusin 2 builds a bridge between ER and mitochondria. Cell 135: 1165-1167, 2008.

36. Scarlatti F, Bauvy C, Ventruti A, et al: Ceramide-mediated macroautophagy involves inhibition of protein kinase $\mathrm{B}$ and up-regulation of beclin 1. J Biol Chem 279: 18384-18391, 2004.

37. Hill CP and Babst M: Structure and function of the membrane deformation AAA ATPase Vps4. Biochim Biophys Acta 1823: 172-181, 2012.

38. Ishibashi K, Fujita N, Kanno E, et al: Atg16L2, a novel isoform of mammalian Atg16L that is not essential for canonical autophagy despite forming an Atg12-5-16L2 complex. Autophagy 7: 1500-1513, 2011.

39. Vogel K, Bentley P, Platt KL and Oesch F: Rat liver cytoplasmic dihydrodiol dehydrogenase. Purification to apparent homogeneity and properties. J Biol Chem 255: 9621-9625, 1980.

40. Glatt HR, Vogel K, Bentley P and Oesch F: Reduction of benzo(a)-pyrene mutagenicity by dihydrodiol dehydrogenase. Nature 277: 319-320, 1979.

41. Penning TM: Dihydrodiol dehydrogenase and its role in polycyclic aromatic hydrocarbon metabolism. Chem Biol Interact 89: 1-34, 1993.

42. Wang HW, Lin CP, Chiu JH, et al: Reversal of inflammationassociated dihydrodiol dehydrogenases (AKR1C1 and AKR1C2) overexpression and drug resistance in nonsmall cell lung cancer cells by wogonin and chrysin. Int J Cancer 120: 2019-2027, 2007.

43. Chen CY, Fang HY, Chiou SH, et al: Sumoylation of eukaryotic elongation factor 2 is vital for protein stability and anti-apoptotic activity in lung adenocarcinoma cells. Cancer Sci 102: 1582-1589, 2011. 